\title{
Sensitivity of the s-process nucleosynthesis in AGB stars to the overshoot model
}

\author{
S. Goriely and L. Siess \\ Institut d'Astronomie et d'Astrophysique, Université Libre de Bruxelles (ULB), CP 226, 1050 Brussels, Belgium \\ e-mail: sgoriely@astro.ulb.ac.be
}

Received 23 June 2017 / Accepted 22 August 2017

\begin{abstract}
Context. $S$-process elements are observed at the surface of low- and intermediate-mass stars. These observations can be explained empirically by the so-called partial mixing of protons scenario leading to the incomplete operation of the $\mathrm{CN}$ cycle and a significant primary production of the ${ }^{13} \mathrm{C}$ neutron source. This scenario has been successful in qualitatively explaining the $s$-process enrichment in AGB stars. Even so, it remains difficult to describe both physically and numerically the mixing mechanisms taking place at the time of the third dredged-up between the convective envelope and the underlying C-rich radiative layer

Aims. We aim to present new calculations of the $s$-process nucleosynthesis in AGB stars testing two different numerical implementations of chemical transport. These are based on a diffusion equation which depends on the second derivative of the composition and on a numerical algorithm where the transport of species depends linearly on the chemical gradient.

Methods. The $s$-process nucleosynthesis resulting from these different mixing schemes is calculated with our stellar evolution code STAREVOL which has been upgraded to include an extended $s$-process network of 411 nuclei. Our investigation focuses on a fiducial $2 M_{\odot},[\mathrm{Fe} / \mathrm{H}]=-0.5$ model star, but also includes four additional stars of different masses and metallicities.

Results. We show that for the same set of parameters, the linear mixing approach produces a much larger ${ }^{13} \mathrm{C}$-pocket and consequently a substantially higher surface $s$-process enrichment compared to the diffusive prescription. Within the diffusive model, a quite extreme choice of parameters is required to account for surface $s$-process enrichment of 1-2 dex. These extreme conditions can not, however, be excluded at this stage.

Conclusions. Both the diffusive and linear prescriptions of the overshoot mixing are suited to describe the $s$-process nucleosynthesis in AGB stars provided the profile of the diffusion coefficient below the convective envelope is carefully chosen. Both schemes give rise to relatively similar distributions of $s$-process elements, but depending on the parameters adopted, some differences may be obtained. These differences are in the element distribution, and most of all in the level of surface enrichment.
\end{abstract}

Key words. nuclear reactions, nucleosynthesis, abundances - stars: AGB and post-AGB

\section{Introduction}

The determination of the surface composition of evolved stars provides important clues to the evolution of their internal structure and their role in the cosmic cycle. In that respect, asymptotic giant branch (AGB) stars form an important class of objects for several reasons. They correspond to the late evolutionary phase of stars with masses between about 1 and $8 M_{\odot}$; they exhibit peculiar chemical patterns at their surface as compared to other red giant stars; and many of them are characterized by strong mass loss (up to $10^{-4} M_{\odot} \mathrm{yr}^{-1}$ ) that ejects the surface material into the interstellar medium, contributing thereby to the galactic chemical evolution.

The abundance peculiarities observed at the surface of AGB stars are the consequence of (i) H-burning at the base of the convective envelope during the so-called hot bottom burning that can lead to a temporary production of ${ }^{7} \mathrm{Li}$ and affects the $\mathrm{CNO}$ isotopes, ${ }^{23} \mathrm{Na}$ or ${ }^{26} \mathrm{Al}$ and (ii) the action of the third dredge-up (denoted hereafter 3DUP) that allows elements synthesized in the He-burning layers to reach the surface. The signatures of the 3DUP are multiple and can explain, for example, the formation of carbon stars, and the surface enrichment in ${ }^{19} \mathrm{~F}, \mathrm{Mg}$ isotopes or heavy elements such as $\mathrm{Ba}$ or $\mathrm{Pb}$ by the so-called slow neutron capture process (for a recent review, see Karakas \& Lattanzio 2014). The ${ }^{13} \mathrm{C}(\alpha, \mathrm{n}){ }^{16} \mathrm{O}$ reaction is currently believed to be the major neutron source in low-mass AGB stars leading to the production of $s$-process elements (Busso et al. 1999). In more massive AGB stars, neutrons can also be released at the bottom of the thermal pulse by the ${ }^{22} \mathrm{Ne}(\alpha, \mathrm{n}){ }^{25} \mathrm{Mg}$ reaction. This production requires high temperatures $\left(T \gtrsim 3.2 \times 10^{8} \mathrm{~K}\right)$ achieved in stars with $M \gtrsim 3-4 M_{\odot}$, depending on the metallicity. The canonical scenario to produce fresh ${ }^{13} \mathrm{C}$ is to invoke the mixing of protons from the envelope into the ${ }^{12} \mathrm{C}$-rich layers during a 3DUP event (e.g., Iben \& Renzini 1982), followed by the incomplete operation of the $\mathrm{CN}$ cycle. The region over which protons are transported is referred to as the partial mixing (PM) zone. Unfortunately, AGB models are still subject to large uncertainties concerning the consistent prediction of both the 3DUP and transport processes. In particular, the 3DUP and PM properties are sensitive to the stellar characteristics (such as stellar mass, metallicity or mass loss rate) and strongly depend on the numerical and physical treatment of the convective boundaries and more specifically on the prescriptions used to account for extra-mixing at the base of the envelope. These additional transport processes are generally attributed to convective overshooting (Herwig et al. 1997), rotationally induced mixing (Herwig et al. 2003; Siess et al. 2004; Piersanti et al. 2013), gravity waves (Denissenkov \& Tout 2003) or mixing driven by magnetic buoyancy (Nucci \& Busso 2014) but remain parametric and poorly constrained. Recent attempts 
to perform two- and three-dimensional hydrodynamical simulation of the He shell convective zone below the AGB envelope (Herwig et al. 2006, 2014; Stancliffe et al. 2011) provide some hints on the nature of the mixing but the resolution and short time scales of the simulations still limit their predictive power.

This paper investigates the impact on the $s$-process nucleosynthesis of the numerical treatment of overshoot mixing at the base of the convective envelope in AGB star. In Sect. 2, we describe the different prescriptions adopted for the implementation of the chemical transport and the nuclear ingredients for the $s$ process reaction network included in our stellar evolution code STAREVOL. The nucleosynthesis of the heavy elements is analyzed in details for a fiducial $2 M_{\odot}[\mathrm{Fe} / \mathrm{H}]=-0.5$ model star up to the end of the AGB phase. In Sect. 3, we study the abundance redistribution of heavy elements $(A \gtrsim 40)$ due to the neutron captures taking place during the core He-burning phase. In Sect. 4, we pay special attention to the $s$-process taking place during the AGB phase with a detailed study of its sensitivity with respect to different overshoot models and different choices of their parameters. We also discuss the final surface abundances for stars of different masses and metallicities in Sect. 5. We finally draw our conclusions in Sect. 6.

\section{Mixing and nucleosynthesis modeling}

The present calculations are based on the stellar evolution code STAREVOL, the description of which can be found in Siess et al. (2000), Siess (2006) and references therein. In our computations, we have used the standard mixing length theory (MLT) with $\alpha=1.75$ and take into account the change in opacity due to the formation of molecules when the star becomes carbon rich, as prescribed by Marigo (2002). The reference solar composition is given by Asplund et al. (2009) which corresponds to a metallicity $Z=0.0134$. For the mass loss rate, we used the Reimers (1975) prescription with $\eta_{\mathrm{R}}=0.4$ from the main sequence up to the beginning of the AGB and then switched to the Vassiliadis \& Wood (1993) rate. The following sections describe in more detail our extended treatments of chemical transport and nuclear burning that have now been consistently included in STAREVOL. We stress that in the present study, we have not considered extra-mixing at the boundaries of the thermal pulse, but only at the base of the convective envelope.

\subsection{An empirical description of the convective overshoot}

The temperature gradient in the convective regions is usually computed by means of the MLT where the radial fluid velocity $\left(v_{\text {conv }}\right)$ depends on the local thermodynamical properties. From this velocity, a diffusion coefficient is defined:

$D_{\text {conv }}=\frac{1}{3} v_{\text {conv }} \Lambda$,

where $\Lambda=\alpha H_{\mathrm{p}}$ is the mixing length, $\alpha$ the MLT parameter and $H_{\mathrm{p}}$ the pressure scale height. This local time-independent theory predicts that at the convective boundary the acceleration of the convective bubbles (but not its velocity) vanishes. However, pushed by their inertia, nothing can prevent the convective cells to overshoot beyond the Schwarzschild limit. This extension of the mixing is usually referred to as overshooting.

In stellar evolution calculations, essentially two standard prescriptions have been proposed to describe convective overshooting. The first and oldest one simply assumes that the convective zone extends over of fraction $d \times H_{\mathrm{p}}^{\mathrm{cb}}$ beyond the Schwarzschild limits, where $H_{\mathrm{p}}^{\mathrm{cb}}$ is the pressure scale height at the convective boundary. The value of $d$ was constrained by fitting the width of massive main sequence stars (e.g., Maeder \& Meynet 1987) and varies between $\approx 0.1-0.25$. The other alternative was proposed by Herwig et al. (1997; see also Herwig 2000) based on the two-dimensional hydrodynamical simulations of Freytag et al. (1996) and assumes a diffusion coefficient in the overshoot region of the form

$D_{\text {over }}=D_{\mathrm{cb}} \exp \left(\frac{-2 z}{f_{\text {over }} H_{\mathrm{p}}^{\mathrm{cb}}}\right)$,

where $D_{\mathrm{cb}}$ is the value of the diffusion coefficient at the base of the convective envelope (as defined by the Schwarzschild criterion), $z$ is the distance from the formal convective boundary and $f_{\text {over }}$ a free parameter describing the efficiency of the diffusive mixing. In a more recent paper, Battino et al. (2016) upgraded this prescription by implementing a double exponential where a second flatter exponential term takes over when the diffusion coefficient has fallen below a threshold value $D_{2} \approx 10^{5} \mathrm{~cm}^{2} / \mathrm{s}$. This second term was fitted on a $3 M_{\odot}$ model to account for the mixing induced by gravity waves. Their formulation corresponds to

$D_{\text {over }}= \begin{cases}D_{\mathrm{cb}} \exp \left(\frac{-2 z}{f_{1} H_{\mathrm{p}}^{\mathrm{cb}}}\right) & \text { if } z<z_{2} \\ D_{2} \exp \left(\frac{-2\left(z-z_{2}\right)}{f_{2} H_{\mathrm{p}}^{\mathrm{cb}}}\right) & \text { if } z>z_{2},\end{cases}$

where the switch between the two regimes occurs at the depth $z_{2}$, determined by imposing that

$D_{2}=D_{\mathrm{cb}} \exp \left(\frac{-2 z_{2}}{f_{2} H_{\mathrm{p}}^{\mathrm{cb}}}\right)$.

In this prescription, $z$ is the distance from the convective boundary and the parameters are $f_{1}, f_{2}$ and $D_{2}$. During the AGB phase, Battino et al. (2016) use the default values of $f_{1}=0.014$, $D_{2}=10^{11} \mathrm{~cm}^{2} / \mathrm{s}$ and $f_{2}=0.25$ at the bottom of the convective envelope.

Essentially, two different approaches can be found in the literature regarding the numerical implementation of the chemical transport. The diffusive mixing scheme (referred to as DM) describes the transport of the nuclear species by a diffusion equation

$\frac{\partial X}{\partial t}=\frac{\partial}{\partial m_{r}}\left[\left(4 \pi r^{2} \rho\right)^{2} D \frac{\partial X}{\partial m_{r}}\right]$,

where $D=D_{\text {conv }}+D_{\text {over }}$ is the sum of the convective and overshoot diffusion coefficients, respectively. However, a diffusive approach of this process may not be very realistic as convection is in essence the advection of convective bubbles.

An alternative algorithm was devised by Sparks \& Endal (1980) and later improved by Chieffi et al. (2001) and Straniero et al. (2006) where mixing is now described by a linear equation coupling the mixing between neighboring layers moving at a velocity $v$ over a time step $\Delta t$. This formulation, which we refer to as linear mixing (LM), is given by

$X_{j}=X_{j}^{0}+\frac{1}{M_{\operatorname{mix}}} \sum_{k}\left(X_{k}^{0}-X_{j}^{0}\right) f_{j, k} \Delta M_{k}$. 


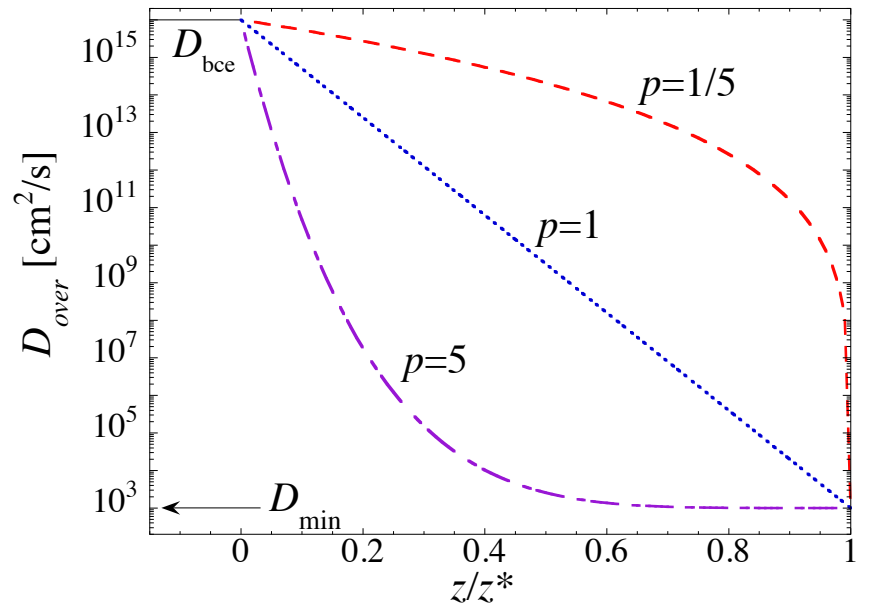

Fig. 1. Profile of the overshoot diffusion coefficient $D_{\text {over }}$ as a function of the reduced depth $z / z^{*}$ for different values of $p$ and assuming $D_{\mathrm{cb}}=$ $10^{16} \mathrm{~cm}^{2} / \mathrm{s}, D_{\min }=10^{3} \mathrm{~cm}^{2} / \mathrm{s}$

In this expression the summation runs overs the whole zone affected by mixing and the superscript 0 refers to abundances prior to the mixing. $\Delta M_{k}$ is the mass of the layer $k$ and the factor

$f_{j, k}= \begin{cases}\Delta t / \tau_{j, k} & \text { if } \Delta t \leq \tau_{j, k}, \\ 1 & \text { if } \Delta t>\tau_{j, k},\end{cases}$

where the mixing turnover time between mesh points $j$ and $k$ is given by

$\tau_{j, k}=\int_{r(j)}^{r(k)} \frac{\mathrm{d} r}{v(r)}=\sum_{i=j}^{k} \frac{\Delta r_{i}}{v_{i}}$,

where $\Delta r_{i}=r_{i+1}-r_{i}$ is the size of the cell. The velocity $v_{i}$ is set to $v_{\text {conv }}$ in the convective layers and below the envelope it decreases exponentially with $v_{i}=v_{\mathrm{cb}} \exp \left(-z / \beta H_{\mathrm{p}}^{\mathrm{cb}}\right)$ where $v_{\mathrm{cb}}$ is the velocity at the base of the convective envelope and $\beta$ a free parameter. We note that this formulation is equivalent to the expression for $D_{\text {over }}$ given by Eq. (2).

In our computation, the total mass of the mixing zone was calculated as $M_{\text {mix }}=\sum_{k} \Delta M_{k} \times f_{k}$ with $f_{k}=\Delta t / \tau_{k}=\Delta t \times v_{k} / \Delta r_{k}$ if $\Delta_{t}<\tau_{k}$, or 1 otherwise. In comparison with the standard expression provided by Straniero et al. (2006) where $M_{\text {mix }}=$ $\sum_{k} \Delta M_{k}$, our formulation presents the advantage of being independent of the limit in the summation, since in the layers where mixing is not present $v_{k}$ and $f_{k}=0$.

In the present study, we have considered both the DM (Eq. (5)) and LM (Eq. (6)) prescriptions and in each case, the adopted mathematical profile for the overshoot diffusion coefficient reads

$D_{\text {over }}=D_{\min } \times\left(\frac{D_{\mathrm{cb}}}{D_{\min }}\right)^{\left(1-z / z^{*}\right)^{p}}$,

where the characteristic length $z^{*}$ is given by $z^{*}=f_{\text {over }} \times H_{\mathrm{p}} \times$ $\ln \left(D_{\mathrm{cb}}\right) / 2, D_{\min }$ is the value of the diffusion coefficient at the boundary $z=z^{*}$ and $p$ is an additional free parameters defining the slope of the exponential decrease of $D_{\text {over }}$ with respect to $z$. Some examples of the $z$-dependence of the overshoot diffusion coefficient are illustrated in Fig. 1 . Note that below $D_{\min }$ we have assumed that $D_{\text {over }}$ drops to zero, in contrast to Straniero et al. (2006) who do not consider a lower limit for $v_{\text {over }}$, hence for
$D_{\text {over }}$ So, with our prescription, the overshoot mixing is only taking place over the radial region defined by $0 \leq z \leq z^{*}$. We note that Eq. (9) is just an extension of Eq. (2) from the original formulation of Herwig et al. (1997) which is recovered by setting $p=1$ and $f_{\text {over }}=0.02$ and $D_{\min }=1 \mathrm{~cm}^{2} / \mathrm{s}$. Similarly, the default parameters used by Straniero et al. (2006) in their simulations correspond to $p=1$ and $f_{\text {over }}=0.2$ and $D_{\min }=1 \mathrm{~cm}^{2} / \mathrm{s}$. We also point out that for a given value of $D_{\mathrm{cb}}$, different parameters $f_{\text {over }}, D_{\text {min }}$ and $p$ can lead to similar profiles of $D_{\text {over }}$ for $z \leq z^{*}$. For example for $D_{\mathrm{cb}}=10^{16} \mathrm{~cm}^{2} / \mathrm{s}$ and $p=1$, the same profile is obtained using $\left(D_{\min }=10^{4} \mathrm{~cm}^{2} / \mathrm{s} ; f_{\text {over }}=0.25\right)$ or $\left(D_{\min }=1 \mathrm{~cm}^{2} / \mathrm{s} ; f_{\text {over }}=0.2\right)$ with the only difference that, in the former case, the diffusion coefficient drops to zero earlier, that is, when $D_{\text {over }}$ reaches $10^{4} \mathrm{~cm}^{2} / \mathrm{s}$.

We also stress that at the time of maximum deepening of the convective envelope during the 3DUP, the size of the CO core is $\approx 0.3-0.4 R_{\odot}$ for our $2 M_{\odot}$ model. This number slightly varies with the mixing parameters (which impact the efficiency of the 3DUP) but remains always of the same order of magnitude as $H_{\mathrm{p}}^{\mathrm{cb}} \approx 0.1 R_{\odot}$. As a consequence, using unrealistically large values of $f_{\text {over }}$ can lead to proton mixing well below the convective envelope and induce a substantial reduction of the $\mathrm{CO}$ core size eventually leading to convergence issues. In practice, values of $f_{\text {over }}$ larger than typically 0.1 can hardly be considered in our model star.

In light of the previous remark, we find that using the double exponential prescriptions of Battino et al. (2016) leads to a complete disappearance of the $s$-process nucleosynthesis. In our stellar models, during the interpulse phase the second exponential term (obtained with the large value of $f_{2}=0.25$ ) was responsible for the transport of the neutron poison ${ }^{14} \mathrm{~N}$ in the region where ${ }^{13} \mathrm{C}$ is burnt. As for rotation (Herwig et al. 2003; Siess et al. 2004), this pollution of the ${ }^{13} \mathrm{C}$ pocket induced by this sustained chemical transport inhibits the production of $s$-process nuclei. We do not understand why this effect does not show up in the computations of Battino et al. (2016).

\subsection{S-process reaction network}

A full nuclear reaction network including 411 nuclei between $\mathrm{H}$ and Po with some 734 nuclear ( $n-, p$-, and $\alpha$-captures), weak (electron captures, $\beta$-decays) and electromagnetic interactions has been implemented in the STAREVOL code. Nuclear reaction rates were taken from the Nuclear Astrophysics Library of the Brussels University ${ }^{1}$ (Arnould \& Goriely 2006), and include the latest experimental and theoretical cross sections through the interface tool NETGEN (Xu et al. 2013a). In particular, all the charged-particle-induced reaction rates of relevance in the $\mathrm{H}$ and He-burning calculations were taken from the NACRE and NACRE-II evaluations (Angulo et al. 1999; Xu et al. 2013b) as well as STARLIB library (Sallaska et al. 2013). When not available experimentally, the cross sections were calculated within the statistical Hauser-Feshbach model with the TALYS reaction code (Goriely et al. 2008). The TALYS calculations were also used systematically to deduce from the laboratory neutron capture cross sections the stellar rates by allowing for the possible thermalization of low-lying states in the target nuclei. We note that at low temperature, the non-thermalization of the isomeric state of ${ }^{26} \mathrm{Al},{ }^{85} \mathrm{Kr},{ }^{115} \mathrm{In},{ }^{176} \mathrm{Lu}$ and ${ }^{180} \mathrm{Ta}$ is introduced explicitly in the reaction network (Käppeler et al. 1989; Nemeth et al. 1994). The temperature- and density-dependent $\beta$-decay and electron capture rates in stellar conditions were taken from

Available at http://www-astro.ulb.ac.be/Bruslib 


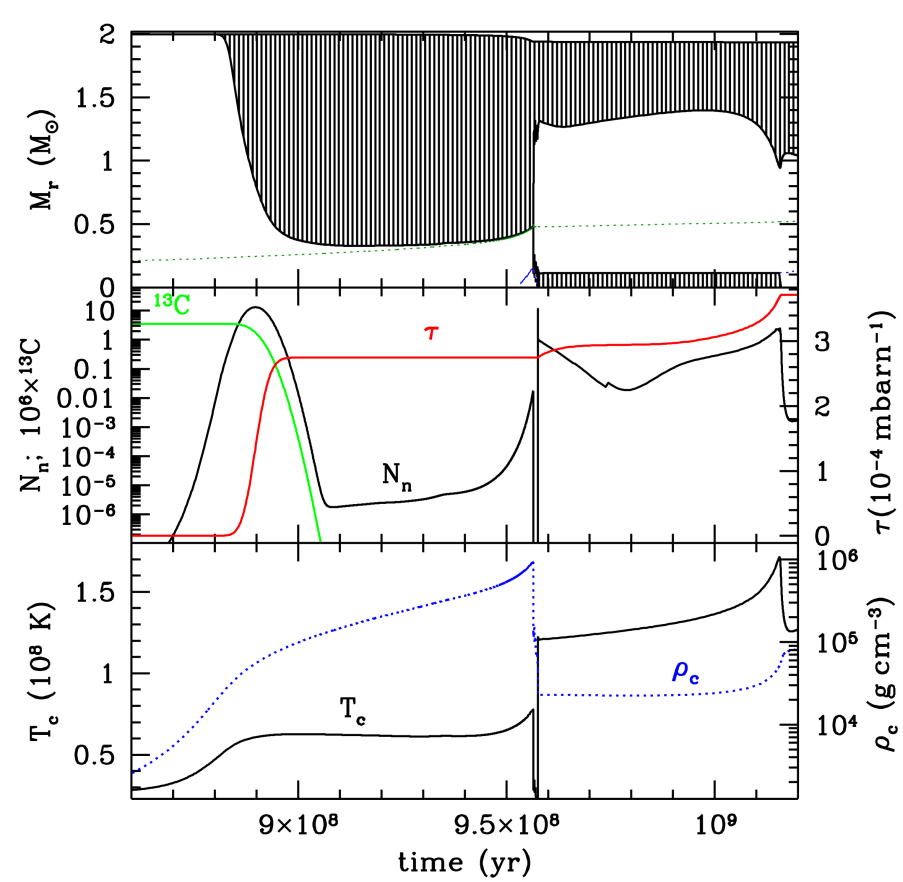

Fig. 2. Upper panel: Kippenhahn diagram of our $2 M_{\odot}[\mathrm{Fe} / \mathrm{H}]=-0.5$ during the first dredge-up and core He burning phases; the green and blue dotted lines delineate the region of maximum nuclear energy production by $\mathrm{H}-$ and He-burning, respectively. Middle panel: evolution of the central neutron density $N_{\mathrm{n}}$, central ${ }^{13} \mathrm{C}$ mass fraction $\left(\times 10^{6}\right)$ and neutron exposure $\tau$. Lower panel: evolution of the central temperature $T_{\mathrm{c}}$ and density $\rho_{\mathrm{c}}$.

Takahashi \& Yokoi (1987) with the update of Goriely (1999). The (n, $\alpha$ ) reactions and $\alpha$-decays are also introduced when relevant, in particular for the $\mathrm{Bi}$ and Po isotopes.

\section{Nucleosynthesis process during core He-burning}

When the star leaves the main sequence and the central temperature reaches $\approx 5 \times 10^{7} \mathrm{~K}$ (for a density $\rho_{\mathrm{c}} \approx 3 \times 10^{4} \mathrm{~g} \mathrm{~cm}^{-3}$ ), the burning of the $\mathrm{CNO}$ by-product ${ }^{13} \mathrm{C}$ via ${ }^{13} \mathrm{C}(\alpha, \mathrm{n}){ }^{16} \mathrm{O}$ produces a first burst of neutrons (Fig. 2). This occurs during the first dredge-up of our $2 M_{\odot}[\mathrm{Fe} / \mathrm{H}]=-0.5$ model star and leads to a maximum neutron density of $N_{\mathrm{n}}=13 \mathrm{~cm}^{-3}$ and a time-integrated neutron exposure of $\tau=\int_{0}^{t} N_{\mathrm{n}} v_{T} \mathrm{~d} t=$ $2.7 \times 10^{-4} \mathrm{mb}^{-1}$, where $v_{T}$ is the most probable relative neutronnucleus velocity at the temperature $T$. The main neutron emission lasts until ${ }^{13} \mathrm{C}$ is depleted at the center and then proceeds at a much lower rate in the contracting shells surrounding the ${ }^{13} \mathrm{C}$-free core. Eventually helium ignites off-center and after a series of shell flashes, convection reaches the center at an age $9.57 \times 10^{8} \mathrm{yr}$. The temperature in the core reaches $1.2 \times 10^{8} \mathrm{~K}$ and can trigger the production of a small amount of neutrons by the activation of the ${ }^{22} \mathrm{Ne}(\alpha, \mathrm{n}){ }^{25} \mathrm{Mg}$ reaction. The neutron exposure increases up to $\tau=3.7 \times 10^{-4} \mathrm{mb}^{-1}$ but remains too small to generate an efficient $s$-process nucleosynthesis. Instead, it is responsible for the re-arrangements of the abundances of specific heavy nuclei. As shown in Fig. 3, elements such as ${ }^{40} \mathrm{~K},{ }^{108} \mathrm{Cd},{ }^{128,130} \mathrm{Xe},{ }^{154} \mathrm{Gd},{ }^{160} \mathrm{Dy},{ }^{170} \mathrm{Yb}$ or ${ }^{198} \mathrm{Hg}$ can be significantly produced with abundances increased by more than $\sim 0.4$ dex with respect to their initial value and others partially destroyed, like ${ }^{107} \mathrm{Ag},{ }^{149} \mathrm{Sm}$ or ${ }^{151} \mathrm{Eu}$. At the temperatures encountered in the stellar center during core He burning, we notice that ${ }^{123} \mathrm{Te},{ }^{176} \mathrm{Lu},{ }^{180} \mathrm{Ta}$ and ${ }^{187} \mathrm{Os}$ become $\beta$-unstable and fully

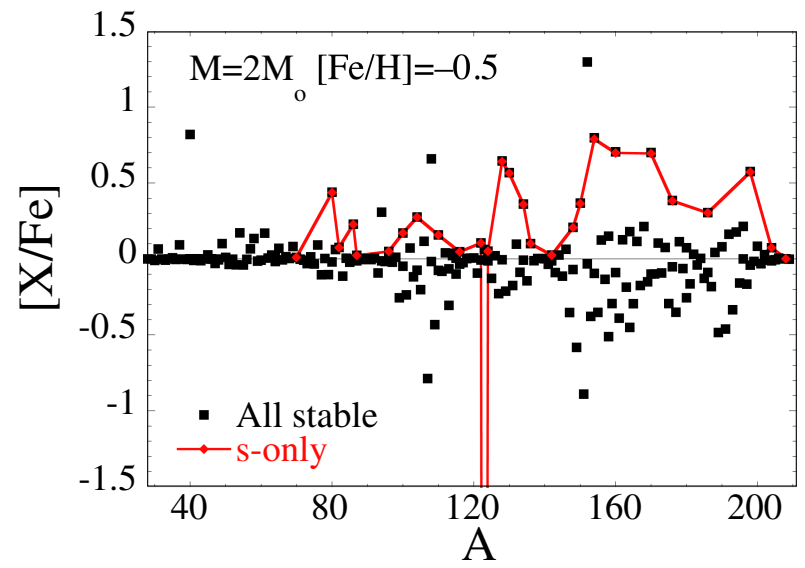

Fig. 3. Final overproduction factors $[\mathrm{X} / \mathrm{Fe}]$ of all stable nuclei with $A \geq$ 30 (full squares) at the center of our $2 M_{\odot}[\mathrm{Fe} / \mathrm{H}]=-0.5$ model star at the end of core helium burning. The s-only nuclei are shown by red diamonds connected by a solid line. The vertical line depicts the total decay of the s-only nucleus ${ }^{123} \mathrm{Te}$.

decay (Takahashi \& Yokoi 1987). When helium is exhausted in the core, the chemical profiles in the central $0.4-0.5 M_{\odot}$ are frozen and the abundances of these heavy nuclei will be part of the future white dwarf. We note that the distributions of overabundances of elements heavier than typically $A=40$ show very small differences between our 2 and $3 M_{\odot}$ stellar models, almost independently of the metallicity.

\section{S-process nucleosynthesis during the AGB phase of a $2 M_{\odot}[\mathrm{Fe} / \mathrm{H}]=-0.5$ model star}

Mixing of the nuclear species beyond the convective boundary in the LM and DM models essentially depends on three parameters: $D_{\min }, p$ and $f_{\text {over }}$. While $f_{\text {over }}$ describes the radial extent over which mixing takes place, the other two parameters $D_{\text {min }}$ and $p$ modify the profile of the diffusion coefficient and thus the distribution of protons in the $\mathrm{C}$-rich region and incidentally the $s$-process nucleosynthesis. In Sect. 4.1 we first study the proton profiles resulting from the overshooting within the LM and DM models and then analyze the production of s-only nuclei during a given interpulse phase (Sect. 4.2) and in the convective pulse (Sect. 4.3). The surface enrichment resulting from the overshoot mixing since the beginning of the AGB phase is discussed in Sect. 4.4.

\subsection{Proton profiles resulting from the treatment of overshoot mixing}

To compare the effects of the two mixing schemes, we began our calculations with the same initial model computed without any extra-mixing to avoid any chemical contamination resulting from previous evolution. We recall that we have not considered overshooting at the convective boundaries of the thermal pulse. The initial model is taken at the time when the convective envelope approaches its deepest extent during the 3DUP following the 8 th thermal pulse of our $2 M_{\odot}[\mathrm{Fe} / \mathrm{H}]=-0.5$ model star. We note that the mass coordinate reached by the convective envelope during the 3DUP depends on the mixing scheme.

We show in Fig. 4 the diffusion coefficients and proton mass fractions below the convective envelope obtained with the LM formalism for different values of the parameters $D_{\min }$ and $p$. As expected, a more efficient transport resulting for example from a 


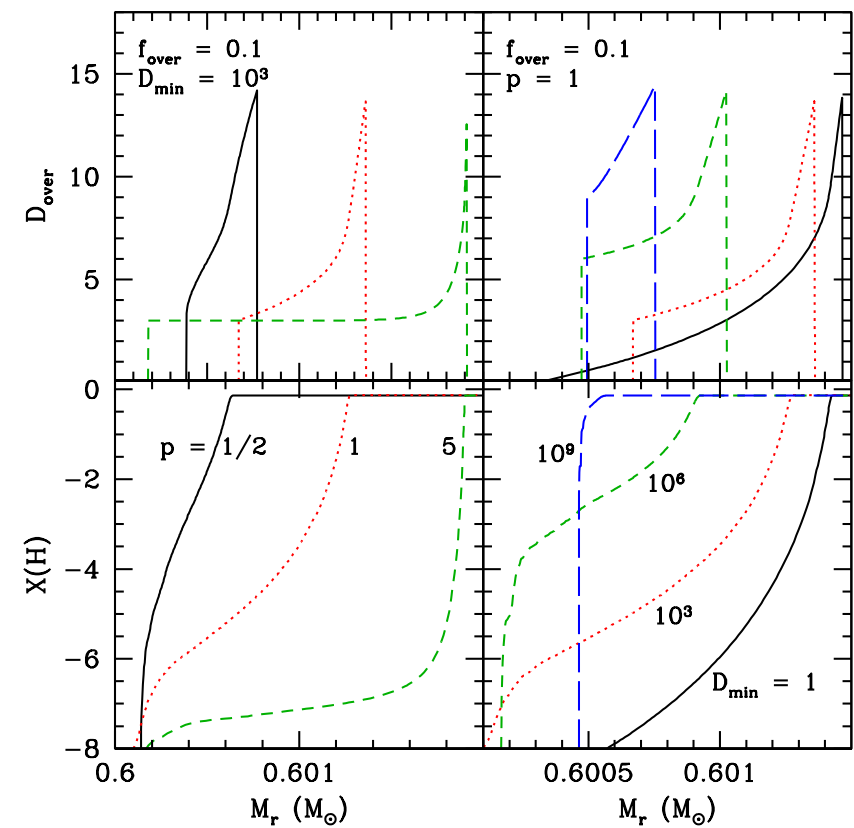

Fig. 4. Left panels: proton mass fraction (bottom panel) and diffusion coefficient (top panel) profiles below the convective envelope resulting from the LM scheme with overshoot parameters $f_{\text {over }}=0.1$, $D_{\min }=10^{3} \mathrm{~cm}^{2} / \mathrm{s}$ and 3 values of $p=1 / 2,1,5$. Right panels: same but for $f_{\text {over }}=0.1, p=1$ and 4 values of $D_{\min }=10^{1.9} \mathrm{~cm}^{2} / \mathrm{s}$. The profiles were taken when the convective envelopes reaches its deepest inward extent. For display convenience, the $D_{\min }=10^{9}$ profiles have been shifted outward by $0.00085 M_{\odot}$.

Table 1. Extent in mass (in unit of $\left.10^{-4} M_{\odot}\right)$ of the PM zone $\left(\Delta M_{\mathrm{pmz}}\right)$ and $s$-process region $\left(\Delta M_{\text {spro }}\right)$ for the LM and DM models and the different adopted values of the parameters $p$ and $D_{\min }\left(\right.$ in $\mathrm{cm}^{2} / \mathrm{s}$ ). In all cases, $f_{\text {over }}=0.1$.

\begin{tabular}{|c|c|c|c|c|c|}
\hline & $p=1$ & $D_{\min }=1$ & $10^{3}$ & $10^{6}$ & $10^{9}$ \\
\hline \multirow[t]{2}{*}{$\Delta M_{\mathrm{pmz}}$} & LM & 2.88 & 5.97 & 7.00 & 0.59 \\
\hline & DM & 0.51 & 0.79 & 2.00 & 0.54 \\
\hline \multirow{2}{*}{$\Delta M_{\text {spro }}$} & LM & 0.52 & 1.04 & 2.62 & 0.02 \\
\hline & DM & 0.10 & 0.15 & 0.41 & 0.0004 \\
\hline & $D_{\min }=10^{3}$ & $p=1 / 2$ & 1 & 5 & \\
\hline \multirow[t]{2}{*}{$\Delta M_{\mathrm{pmz}}$} & LM & 4.12 & 5.97 & 0.87 & \\
\hline & DM & 1.25 & 0.79 & 0.18 & \\
\hline \multirow[t]{2}{*}{$\Delta M_{\text {spro }}$} & LM & 1.06 & 1.04 & 0.15 & \\
\hline & $\mathrm{DM}$ & 0.23 & 0.15 & 0.03 & \\
\hline
\end{tabular}

higher value of $D_{\min }$ or a flatter profile obtained with a lower $p$ leads to a deeper 3DUP. Following Goriely \& Mowlavi (2000), we defined the extent of the PM zone $\Delta M_{\mathrm{pmz}}$ as the mass range over which the hydrogen mass fraction decreases from $X(\mathrm{H})=$ 0.5 (close to its envelope value) to $X(\mathrm{H})=10^{-5}$. As shown in Table $1, \Delta M_{\mathrm{pmz}}$ varies between $\approx 6 \times 10^{-5} M_{\odot}$ (for $p=1, D_{\min }=$ $\left.10^{9} \mathrm{~cm}^{2} / \mathrm{s}\right)$ to almost $7 \times 10^{-4} M_{\odot}\left(\right.$ for $\left.p=1, D_{\text {min }}=10^{6} \mathrm{~cm}^{2} / \mathrm{s}\right)$. We also note that with increasing values of $D_{\min }$, the $\mathrm{H}$ mass fraction remains relatively high in the mixing region and then drops rapidly.

In the DM case (Fig. 5), the extent of the PM zone is systematically smaller by a factor of between three and eight compared with the values obtained with the LM prescription, except in the rather extreme case of $D_{\min }=10^{9}$. Although this study has been restricted to a specific interpulse phase, the derived properties (extent of the mixing, $\mathrm{H}$ profiles) remain general and weakly

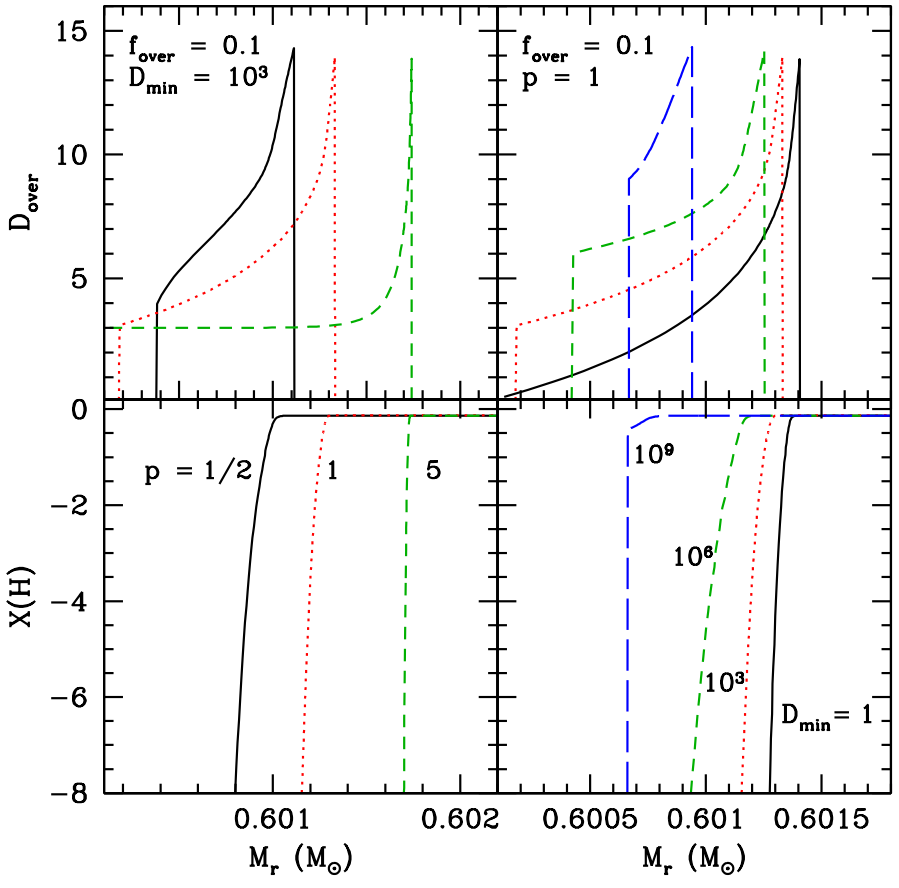

Fig. 5. As Fig. 4 but for the diffusive treatment (DM) of overshooting.

dependent on mass or composition as long as the background stellar stratification is not significantly affected (Herwig et al. 2007).

\subsection{S-process nucleosynthesis during an interpulse}

In this section we analyze the overproduction factors $[\mathrm{X} / \mathrm{Fe}]$ of the 28 s-only nuclei at the end of the eighth interpulse of our $2 M_{\odot}[\mathrm{Fe} / \mathrm{H}]=-0.5$ model star resulting from the two different mixing schemes (DM and LM). The abundances are determined by averaging the chemical composition over a mass range of $0.01 M_{\odot}$ between $M_{r}=0.595$ and $0.605 M_{\odot}$. We recall that the same initial stellar structure is considered in all cases (the fully consistent stellar evolution sequences are presented in Sects. 4.4 and 5).

The results of our exploration of the mixing parameter space are displayed in Figs. 6 and 7 for the LM and DM schemes, respectively. The first obvious observation is the similarity of the distribution of $s$-elements between all the models, except may be for the extreme case $p=1, D_{\min }=10^{9} \mathrm{~cm}^{2} / \mathrm{s}$. The main difference is the level of overproduction.

During the interpulse phase, the $s$-process nucleosynthesis is known to take place in a relatively small radiative region where the proton-to- ${ }^{12} \mathrm{C}$ ratio ranges between 0.06 and 0.6 , although in more metal poor stars with $[\mathrm{Fe} / \mathrm{H}] \lesssim-1$, layers with ratios as low as 0.002 also contribute (Goriely \& Mowlavi 2000). For an He intershell carbon mass fraction $X\left({ }^{12} \mathrm{C}\right)=0.2$, the neutron production will mainly occur in layers where the proton mass fraction $X(\mathrm{H}) \simeq 10^{-3}-10^{-2}$, while for larger values of $X(\mathrm{H}) \simeq 10^{-2}-10^{-1}$ an efficient production of ${ }^{19} \mathrm{~F}$ and ${ }^{23} \mathrm{Na}$ is expected. As discussed in Goriely \& Mowlavi (2000), the detailed profile of protons in this region dictates the subsequent $s$-process nucleosynthesis (see in particular their Fig. 3), so the extent of the PM zone $\Delta_{\mathrm{pmz}}$ as defined in Sect. 4.1 is only a qualitative and approximate indicator of the $s$-process efficiency. For example, the value of $\Delta_{\mathrm{pmz}}$ picked in Table 1 for the DM scheme is similar between the models $D_{\min }=1$ and $D_{\min }=10^{9}$ 


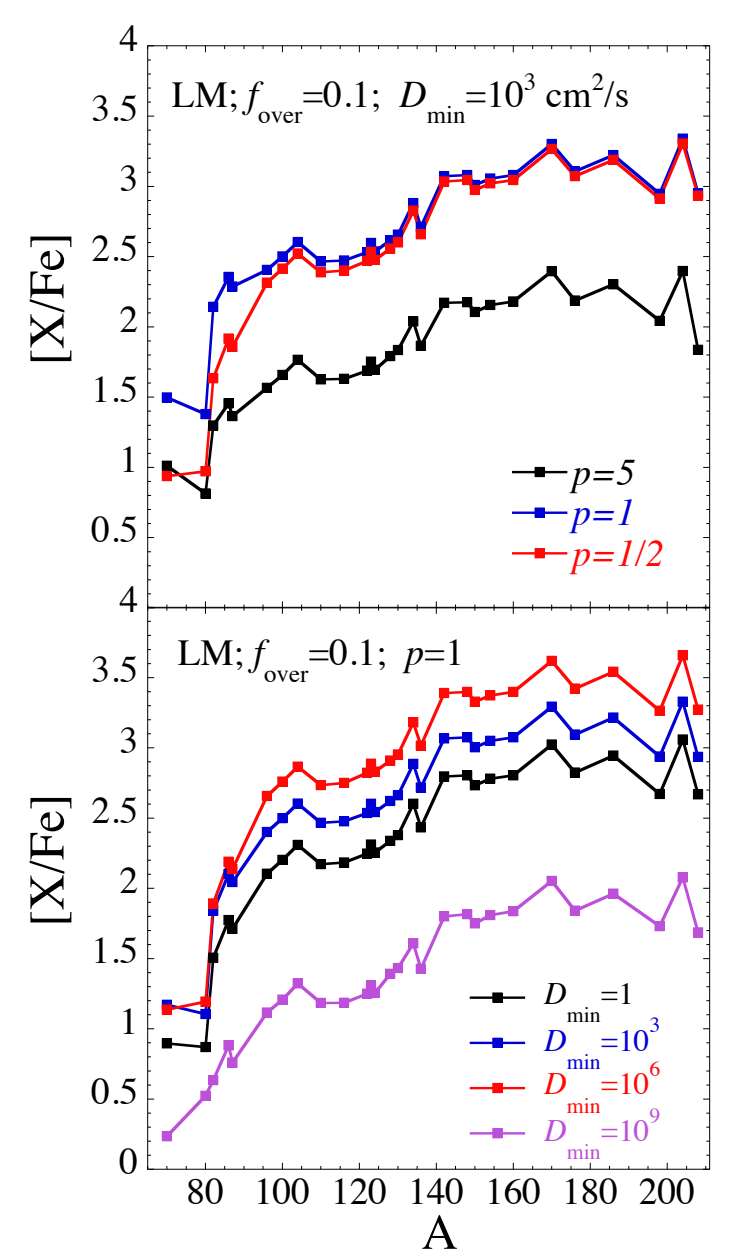

Fig. 6. Overabundance distributions $[\mathrm{X} / \mathrm{Fe}]$ for the 28 s-only nuclei inside a region of $0.01 M_{\odot}$ around $M_{r}=0.595-0.605 M_{\odot}$ at the end of the 8th interpulse of our $2 M_{\odot}[\mathrm{Fe} / \mathrm{H}]=-0.5$ model star. The results are shown for different LM conditions, i.e. different values of $p$ (upper panel) or $D_{\min }\left(\right.$ in $\mathrm{cm}^{2} / \mathrm{s}$; lower panel); in all cases, $f_{\text {over }}=0.1$ is assumed.

but the resulting $s$-process enrichment (Fig. 7) is significantly different. For this reason, we defined a new indicator $\Delta M_{\text {spro }}$ corresponding to the mass extent where $10^{-3} \leq X(\mathrm{H}) \leq 10^{-2}$. An inspection of the numbers presented in Table 1 indicates that $\Delta M_{\mathrm{pmz}}$ and $\Delta M_{\mathrm{spro}}$ are quite different but strongly correlated. There are, however, two exceptions, one is related to the extreme case $D_{\min }=10^{9} \mathrm{~cm}^{2} / \mathrm{s}$ mentioned previously and the second to the LM cases $p=1 / 2$ and $p=1$ where the nucleosynthesis is very similar. Comparing Figs. 6, 7, and Table 1 indicates that the highest overproduction factors are obtained for the largest values of $\Delta M_{\text {spro. }}$. In the LM case, $\Delta M_{\text {spro }}$ reaches a maximum of $\sim 3 \times 10^{-4} M_{\odot}$ for $p=1 / 2-1$ and $D_{\min } \approx 10^{3}-10^{6} \mathrm{~cm}^{2} / \mathrm{s}$, leading to a significant production of $s$-process nuclei (Fig. 6). In contrast, in the DM case, $\Delta M_{\text {spro }}$ never exceeds $3 \times 10^{-5} M_{\odot}$, that is, about an order of magnitude lower than in the LM case. As shown in Fig. 7, the overall overproduction factors are indeed a factor of between five and ten lower than in the LM model. This significant enrichment in $s$-process elements by the LM scheme was also reported by Cristallo et al. (2009).

\subsection{Convective nucleosynthesis in the thermal pulse}

The distribution of $s$-elements resulting from the radiative interpulse nucleosynthesis, as described in Sect. 4.2, may still be

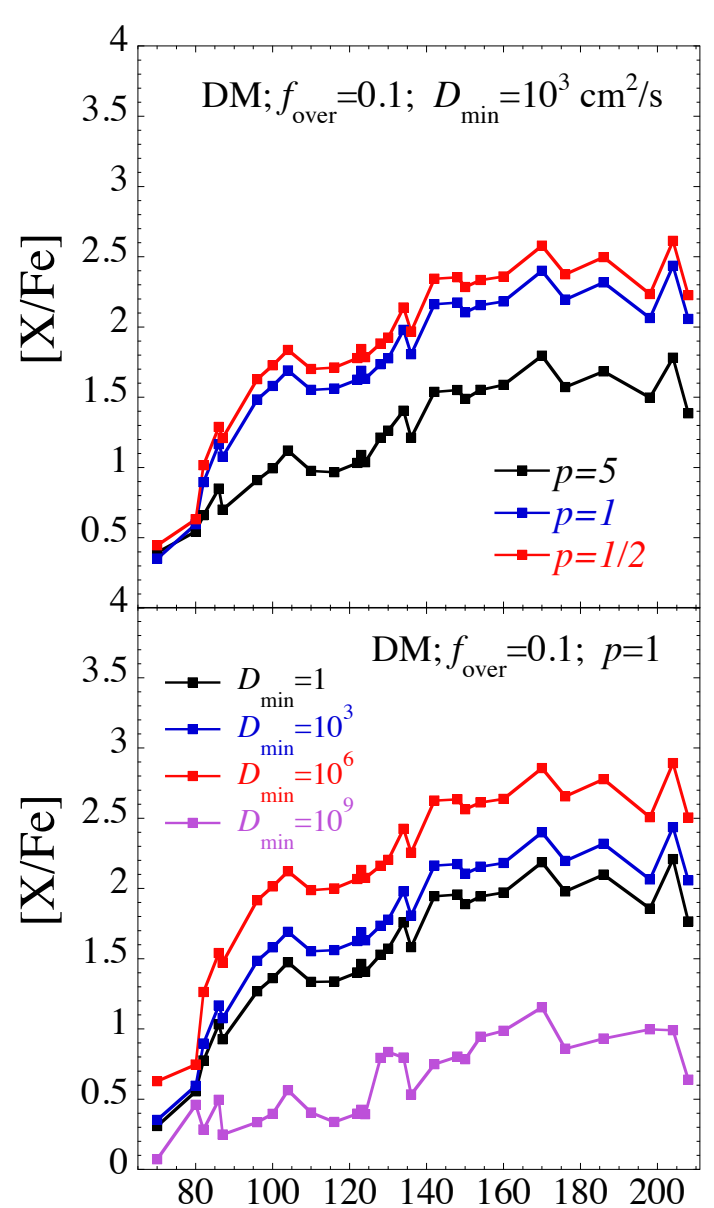

Fig. 7. As Fig. 6 for the $s$-process distributions obtained with the DM model.

affected by the second neutron irradiation taking place in the pulse-driven convective region, at least if the temperature $T_{\mathrm{p}}$ at its base is high enough to activate the ${ }^{22} \mathrm{Ne}(\alpha, \mathrm{n}){ }^{25} \mathrm{Mg}$ reaction. $T_{\mathrm{p}}$ depends on the core mass and is known to increase with increasing pulse number, stellar mass or decreasing metallicities and ranges from $1.5 \times 10^{8} \mathrm{~K}$ to above $3.5 \times 10^{8} \mathrm{~K}$. In our $2 M_{\odot}[\mathrm{Fe} / \mathrm{H}]=-0.5$ model star, $T_{\mathrm{p}}$ reaches $3 \times 10^{8} \mathrm{~K}$ in the last thermal pulses, so only few neutrons are produced. As seen in Fig. 8, the impact of the convective pulse nucleosynthesis on the abundance distribution of s-only nuclei is negligible. However, locally, the abundances of some nuclei, such as ${ }^{93} \mathrm{Nb}$, ${ }^{113} \mathrm{In},{ }^{176} \mathrm{Lu}$ or ${ }^{187} \mathrm{Os}$ may be modified through the impact of $T$-dependent $s$-process branching during the neutron irradiation (Takahashi \& Yokoi 1987). In the mass and metallicity range considered in this study, none of the ${ }^{13} \mathrm{C}$ produced by the PM of protons survives the interpulse phase. Therefore, it remains to be checked if, in low-mass metal-poor stars $\left(M \lesssim 1.5 M_{\odot}\right.$, $[\mathrm{Fe} / \mathrm{H}] \lesssim-2)$, fresh ${ }^{13} \mathrm{C}$ is ingested in the convective pulse, as found in previous studies (Lugaro et al. 2012).

\subsection{Nucleosynthetic yields}

In this section, we present the nucleosynthesis yields of a $2 M_{\odot}[\mathrm{Fe} / \mathrm{H}]=-0.5$ model computed with the LM and DM mixing formalisms. For these simulations, the same mixing parameters are used, namely $D_{\text {min }}=10^{3} \mathrm{~cm}^{2} / \mathrm{s}, p=1$ and $f_{\text {over }}=0.1$, and applied since the beginning of the AGB phase. The thermal pulse-AGB evolution is similar in both cases and leads to the 
S. Goriely and L. Siess: Sensitivity of the $s$-process to the overshoot model

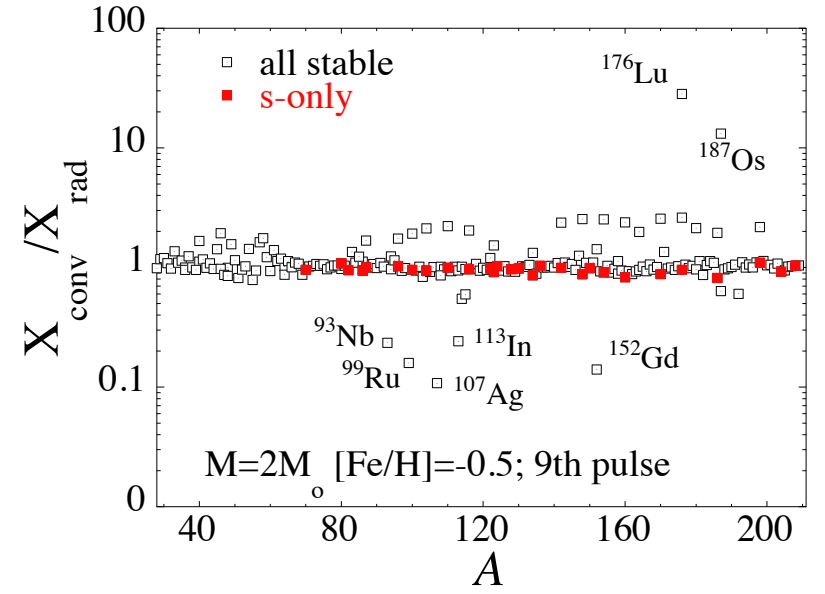

Fig. 8. Ratio of the nuclei mass fraction after $\left(X_{\text {conv }}\right)$ and before $\left(X_{\text {rad }}\right)$ the 9th thermal pulse of our $2 M_{\odot}[\mathrm{Fe} / \mathrm{H}]=-0.5$ model star. Open and filled squares represent stable and s-only nuclei, respectively.

occurrence of ten thermal pulses and 8 3DUP. The surface overabundance of s-only nuclei obtained at the end of the computation is presented in Fig. 9. Although the two distributions are relatively similar, the overproduction obtained with the DM is about eight times smaller than with the LM prescription. Again, this has to be ascribed to the smaller mass of the PM zone in the DM formulation. While the LM prescription gives rather satisfactory overproduction factors of the order of $1.5 \mathrm{dex}$, they do not exceed 0.9 dex in the DM case. The latter would consequently face problems explaining the large surface abundances of some intrinsic $s$-process-rich C-stars that show for example $[\mathrm{Ba} / \mathrm{Fe}]$ as high as 1.65 (Zamora et al. 2009).

In the specific DM case, we have investigated the impact of the mixing parameters on the final surface enrichment. The results for nine sets of mixing parameters, that is, $D_{\min }=10^{3}, 10^{6}$, and $10^{9} \mathrm{~cm}^{2} / \mathrm{s}, p=1 / 5,1,5$ are shown in Fig. 10. In all these models, $f_{\text {over }}=0.1$ and overshooting is activated at the beginning of the AGB phase. Most cases lead to a rather low surface enrichment, except for the case $D_{\text {min }}=10^{6} \mathrm{~cm}^{2} / \mathrm{s}, p=1$ where an enrichment of the order of 1 dex is achieved and for the set $D_{\text {min }}=10^{9} \mathrm{~cm}^{2} / \mathrm{s}, p=5$ which gives rise to an overproduction of about 2 dex for the heaviest s-only nuclei comparable to the one obtained with the LM prescription. In this latter quite extreme case, a relatively flat proton profile with $X(\mathrm{H}) \simeq 10^{-3}-10^{-2}$ is achieved over a large fraction of the PM zone, leading to a significant production of $s$-process nuclei during the interpulse phase.

\section{S-process in stars of different masses and metallicities}

As shown in Sect. 4, the abundance distribution of s-nuclei is rather insensitive to the mixing scheme and parametrisation but the production factors strongly depend on its mathematical formulation. The LM model consistently produces significant surface enrichment while the DM approach requires a fine tuning of the underlying parameters to achieve a similar result. Assuming the production of $s$-elements is similar, it remains to be seen if the overall abundance distributions obtained using both models are comparable for stars with different initial masses and metallicities. The parameters used are $D_{\min }=10^{3} \mathrm{~cm}^{2} / \mathrm{s}$, $p=1$ for the LM scheme and $D_{\min }=10^{9} \mathrm{~cm}^{2} / \mathrm{s}, p=5$ for the DM ( $f_{\text {over }}=0.1$ is taken in both cases). The high value of

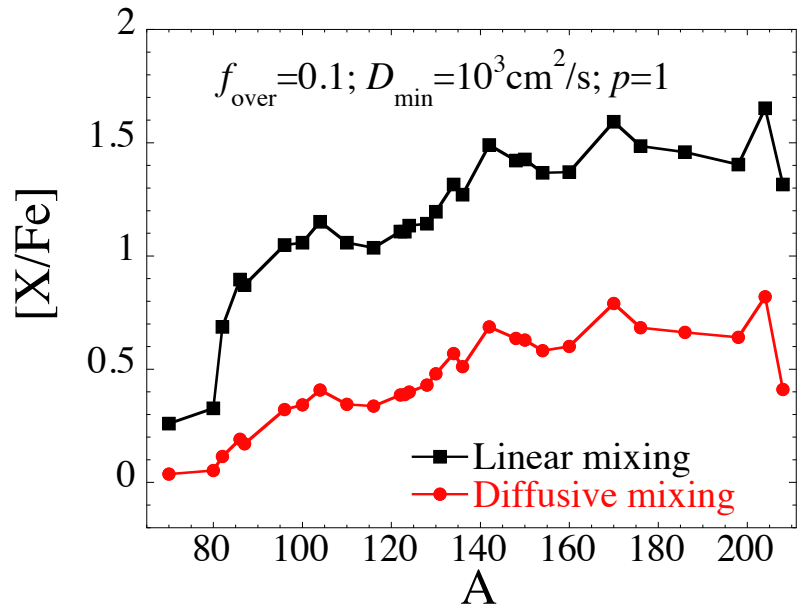

Fig. 9. Surface overabundance distributions $[\mathrm{X} / \mathrm{Fe}]$ for the $28 \mathrm{~s}$-only nuclei at the end of the AGB phase of a $2 M_{\odot}[\mathrm{Fe} / \mathrm{H}]=-0.5$ model star resulting from the LM and DM schemes. In both cases, $D_{\min }=$ $10^{3} \mathrm{~cm}^{2} / \mathrm{s}, p=1$ and $f_{\text {over }}=0.1$.

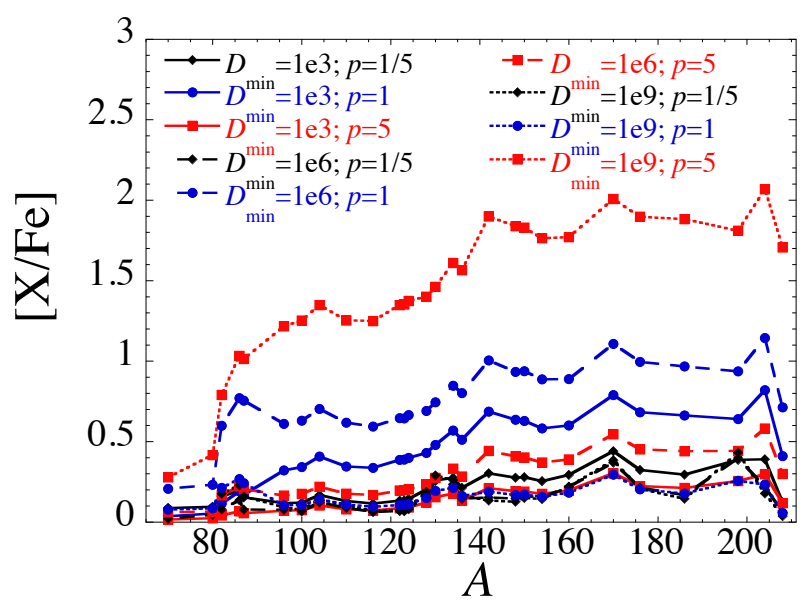

Fig. 10. Surface overabundance distributions $[\mathrm{X} / \mathrm{Fe}]$ for the 28 s-only nuclei at the end of the AGB phase of a $2 M_{\odot}[\mathrm{Fe} / \mathrm{H}]=-0.5$ model star computed with the DM overshoot formalism and 9 different sets of mixing parameters, i.e. $D_{\min }=10^{3}, 10^{6}$, and $10^{9} \mathrm{~cm}^{2} / \mathrm{s}, p=1 / 5,1,5$ and $f_{\text {over }}=0.1$.

$D_{\text {min }}=10^{9} \mathrm{~cm}^{2} / \mathrm{s}$ was chosen, so that similar surface enrichments are produced between both schemes, making the comparison easier. We are aware that such a high value is difficult to justify on physical grounds but considering a value lower than $D_{\text {min }}=10^{7} \mathrm{~cm}^{2} / \mathrm{s}$ would reduce the PM zone significantly, leading to a negligible $s$-process production. Figure 11 shows the mass extent of the PM zone $\Delta M_{\mathrm{pmz}}, s$-process region $\Delta M_{\text {spro }}$, as defined in Sect. 4.2, and the mass $\Delta M_{3 D U P}$ of 3DUP material as a function of the pulse number, for a $2 M_{\odot}$ model computed with three different initial metallicities $[\mathrm{Fe} / \mathrm{H}]=0,-0.5$ and -1 . The decline of the PM and $s$-process zones with the pulse number is a consequence of the growing core mass which produces a strong gravitational pull and thus a higher compression of the layers located below the convective envelope (see also Cristallo et al. 2009). While the LM models give rise to a rather smooth and constant decrease of the PM and $s$-process zones, irrespective of the metallicity, significantly larger variations are found with the DM prescription. Again, one can see the correlation between the size of the $s$-process zone (Fig. 11) and the surface overproduction factors (Fig. 12), except to some extent for the $[\mathrm{Fe} / \mathrm{H}]=-1$ 


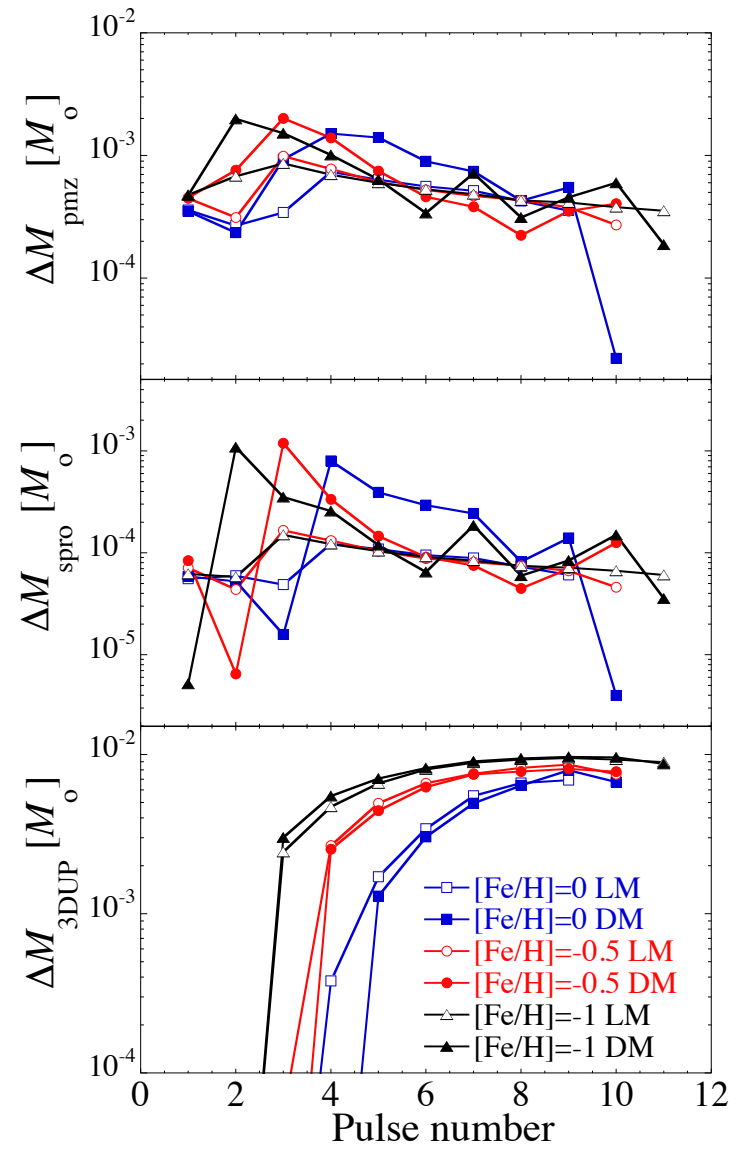

Fig. 11. Upper panel: extent of the PM zone $\Delta M_{\mathrm{pmz}}$ in a $2 M_{\odot}$ model as a function of the pulse number for 3 different initial metallicities; $[\mathrm{Fe} / \mathrm{H}]=0,-0.5$ and -1 . The LM scheme uses $D_{\min }=10^{3} \mathrm{~cm}^{2} / \mathrm{s}, p=1$ and $f_{\text {over }}=0.1$ and the DM case $D_{\text {min }}=10^{9} \mathrm{~cm}^{2} / \mathrm{s}, p=5$ and $f_{\text {over }}=$ 0.1 . Middle panel: same but for the mass of the $s$-process zone $\Delta M_{\text {spro }}$. Lower panel: same but for the dredged-up mass $\Delta M_{3 \mathrm{DUP}}$

model star where the $10^{-4} \lesssim X(\mathrm{H}) \lesssim 10^{-3}$ mass range, neglected in the definition of $\Delta M_{\text {spro }}$, may contribute to the $s$-process nucleosynthesis (Goriely \& Mowlavi 2000). As far as the dredgeup mass is concerned, both prescriptions yield rather similar values, at least when the 3DUP efficiency has reached its asymptotic value, that is, after the first three to five thermal pulses depending on the stellar metallicity (Fig. 11).

The sensitivity of the $s$-process production on the mixing scheme and initial composition is illustrated in Fig. 12. As can be seen, the final surface enrichment between the two mixing schemes mainly differs in terms of overproduction factors, but also in terms of isotopic and elemental distributions. With our specific choice of parameters, the DM scheme yields similar or even higher $s$-process overproduction factors than the standard LM parametrization. It remains, however, difficult to reach surface enrichments larger than typically 2 dex. At solar metallicity the $s$-process surface enrichment is relatively weak, despite the fact that the extent of the $s$-process zone (Fig. 11) is relatively similar to the other model stars with lower metallicities. This is essentially due to the smaller number and relatively shallower 3DUP episodes found in solar metallicity stars. While six 3DUP are predicted in our $2 M_{\odot}[\mathrm{Fe} / \mathrm{H}]=0$ model star, nine are found for a metallicity of $[\mathrm{Fe} / \mathrm{H}]=-1$. In addition, in the last five thermal pulse-interpulse sequences, the 3DUP of the solar metallicity star drags from $5 \%$ to $25 \%$ of the thermal pulse material into

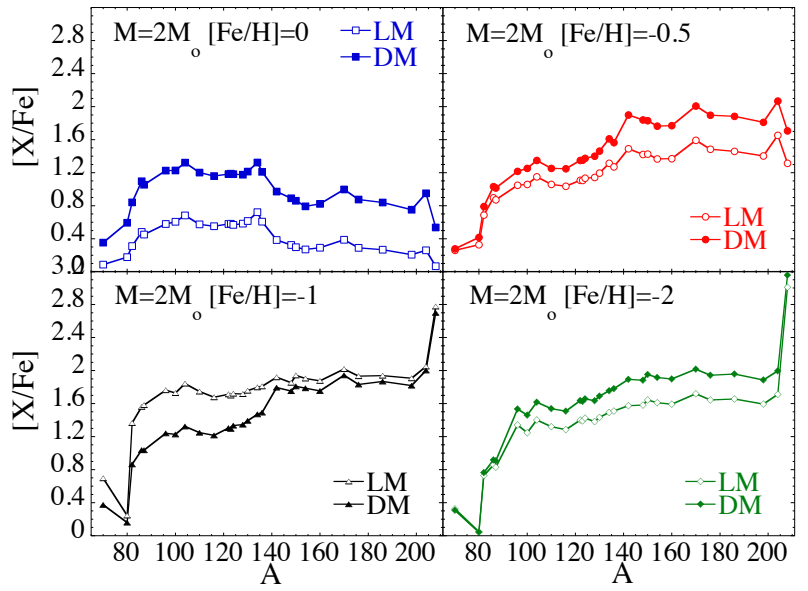

Fig. 12. Dependence on stellar metallicity of the final surface overabundance distributions $[\mathrm{X} / \mathrm{Fe}]$ of the 28 s-only nuclei at the surface of a $2 M_{\odot}$ model resulting from the LM scheme with $D_{\min }=10^{3} \mathrm{~cm}^{2} / \mathrm{s}$, $p=1$ and the DM formulation with $D_{\min }=10^{9} \mathrm{~cm}^{2} / \mathrm{s}, p=5$. In both cases, $f_{\text {over }}=0.1$ is taken. Four metallicities $[\mathrm{Fe} / \mathrm{H}]=0,-0.5,-1$ and -2 have been considered.

the envelope, while the 3DUP efficiency reaches more than $35 \%$ in the $[\mathrm{Fe} / \mathrm{H}]=-1$ model star. This pattern is found to be qualitatively independent of the adopted overshoot model, although quantitatively some minor differences are found.

Some differences in the element distribution can also be found. In particular, when compared with the LM scheme, the DM models tends to give rise to a lower production of light $s$ elements with respect to the heavy $s$-elements, as seen in Figs. 12 and 13, and in Table 2. While this difference is marginal in the solar metallicity star, this is particularly clear in the $2 M_{\odot}$, $[\mathrm{Fe} / \mathrm{H}]=-1$ model star, where a lower $90 \lesssim A \lesssim 140$ distribution by almost 0.5 dex is found with the DM model compared to the LM formulation. The corresponding observational [hs/ls] index, where hs describes the heavy $s$-elements ( $\mathrm{Ba}, \mathrm{La}$ and/or $\mathrm{Nd}$ ) abundance and ls the light $s$-elements ( $\mathrm{Sr}, \mathrm{Y}$ and/or $\mathrm{Zr}$ ) abundance, can differ by up to 0.2 dex (Table 2). The low production of light $s$-elements with the DM model is due to the specific profile of the diffusion coefficient obtained with the large $p=5$ and $D_{\min }=10^{9} \mathrm{~cm}^{-2} / \mathrm{s}$ values (see Figs. 1 and 5). With these parameters, more weight is given to the layers with proton abundances close to $X(\mathrm{H}) \simeq 10^{-3}$ at the expense of $X(\mathrm{H}) \simeq 10^{-2}$, and as a consequence the production of light elements is reduced with respect to heavy ones (see in particular Fig. 3 of Goriely \& Mowlavi 2000).

We also notice that with decreasing metallicity, the production of heavier elements is favored as a consequence of the lower abundance of iron seed nuclei, hence a larger neutronto-seed ratio. We note the systematic overproduction of $\mathrm{Pb}$ at $[\mathrm{Fe} / \mathrm{H}] \leq-1$ which accounts for Pb-stars (Van Eck et al. 2001), but none of the models studied here can explain some $s$-process enriched C-enhanced metal-poor stars (Bisterzo et al. 2011; Piersanti et al. 2013) as well as low-metallicity post-AGB stars (e.g., De Smedt et al. 2014, 2016) that are poorly enriched in $\mathrm{Pb}$.

Finally, the effect of varying the initial mass is illustrated in Fig. 13. As confirmed by Table 2, these calculations indicate that, with increasing stellar mass, the [hs/ls] ratio decreases, essentially because of some additional production of light $s$-elements in hotter thermal pulses. While in our $2 M_{\odot}[\mathrm{Fe} / \mathrm{H}]=-0.5$ model star, $T_{\mathrm{p}}$ does not exceed $3 \times 10^{8} \mathrm{~K}$, in the $3 M_{\odot}$ model 

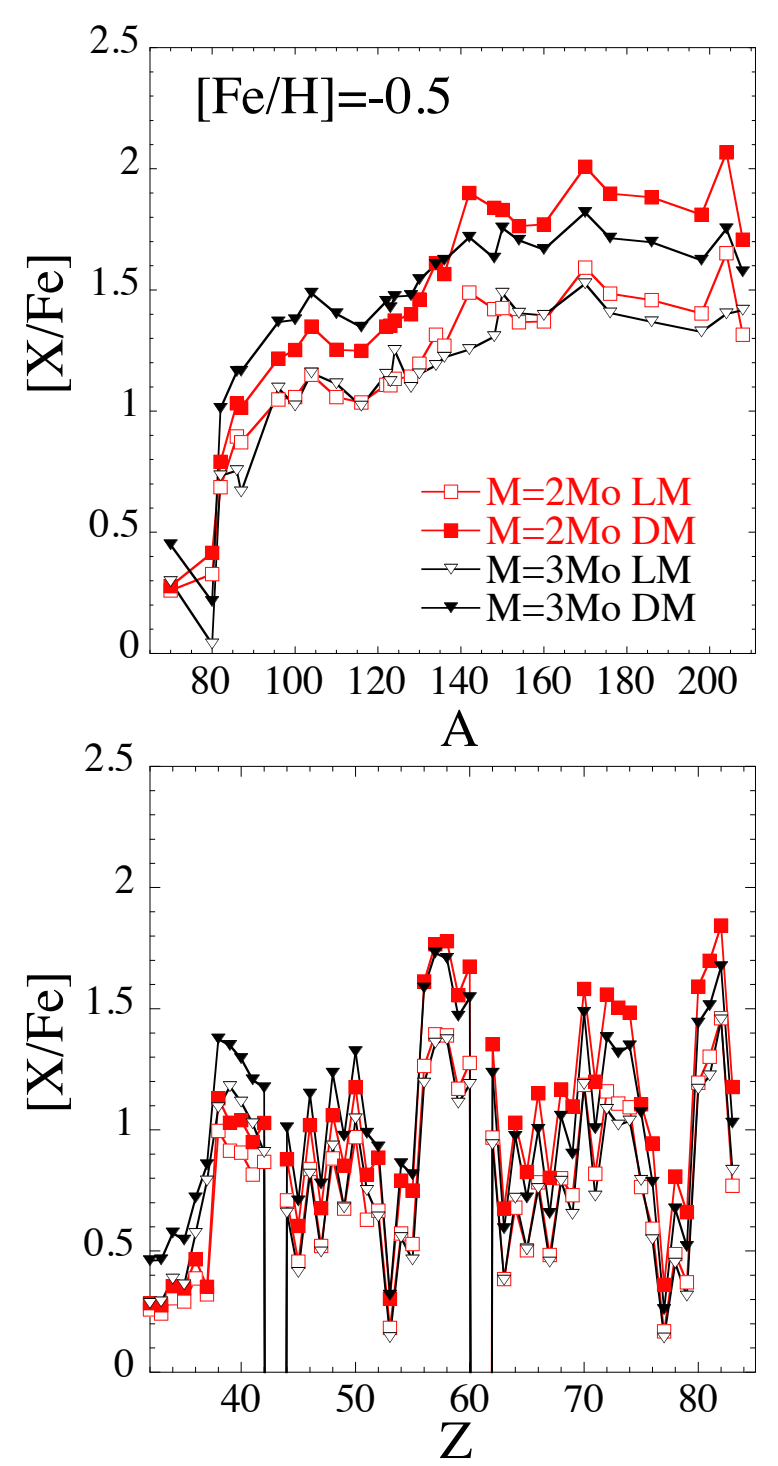

Fig. 13. Top panel: as Fig. 12 for the dependence on the stellar mass of the final surface overabundance distribution of s-only nuclei. Stars of 2 and $3 M_{\odot}$ with a metallicity $[\mathrm{Fe} / \mathrm{H}]=-0.5$ are considered. Lower panel: same as the left panel for the final surface elemental distribution of all elements above $\mathrm{Ge}$.

it reaches $3.5 \times 10^{8} \mathrm{~K}$, allowing for the activation of the ${ }^{22} \mathrm{Ne}(\alpha$, n) ${ }^{25} \mathrm{Mg}$ reaction in the convective thermal pulse. The [hs/ls] index is found to be smaller by $0.3(0.2)$ dex in the $3 M_{\odot}$ star with respect to the $2 M_{\odot}$ star when adopting the LM (DM) model. Future calculations will explore stellar models within a wider range of masses and metallicities.

Independently of this work, Buntain et al. (2017) investigated the sensitivity of the $s$-process nucleosynthesis to the proton profile and mass extent of the PM zone. Their approach differs from ours as they perform post-processing calculations in which they impose the proton profile at the time of the 3DUP and the extent of the PM zone $\Delta M_{\mathrm{pmz}}$. These authors find that the resulting $s$-process abundance distribution remains weakly (by more or less 0.2 dex) dependent on the shape of the mixing function, except at low metallicity $(Z=0.0001)$ where the $\mathrm{Pb}$ abundance can be significantly affected. These results are consistent with those presented here, except for the $\mathrm{Pb}$ production in metal poor-stars that always remain relatively high in our calculations.
Table 2. Surface $[\mathrm{hs} / \mathrm{ls}]$ index deduced from the $[\mathrm{La} / \mathrm{Y}]$ ratio at the end of the evolution of the five model stars studied here and for both LM and DM schemes.

\begin{tabular}{cccc}
\hline \hline$M\left[M_{\odot}\right]$ & {$[\mathrm{Fe} / \mathrm{H}]$} & $\mathrm{LM}$ & $\mathrm{DM}$ \\
\hline 2 & 0. & 0.00 & 0.05 \\
2 & -0.5 & 0.48 & 0.58 \\
2 & -1 & 0.39 & 0.52 \\
2 & -2 & 0.36 & 0.51 \\
3 & -0.5 & 0.18 & 0.38 \\
\hline
\end{tabular}

\section{Conclusions}

Assuming overshooting below the convective envelope of an AGB stars can be described by an exponential decrease of the diffusion coefficient or equivalently of the convective velocity, we analyzed the impact of two different numerical modeling of the chemical transport on the resulting $s$-process nucleosynthesis. We have shown that for the same set of parameters, both descriptions give a rather similar abundance distribution of sonly nuclei (though some differences are found in the relative production of light to heavy $s$-elements), but the surface enrichment can differ drastically. As found by Cristallo et al. (2009), the linear algorithm leads to the formation of a PM zone that can easily account for large surface $s$-process enrichments. On the other hand, the DM approach requires a quite extreme set of parameters producing diffusion profiles that sharply drop to zero below a threshold value of $D_{\min } \sim 10^{7}-10^{9} \mathrm{~cm}^{2} / \mathrm{s}$. At this stage, it is however not possible to exclude this set of parameters, nor to favor one prescription over the other. Both schemes are able to phenomenologically simulate the PM of protons inside the C-rich layers. Our exploration of the parameters attests of the difficulty to find a choice of parameters that leads to surface enrichments significantly larger than typically 2 dex for the bulk of $s$-elements. However, it remains unclear if stars of different masses and metallicities are characterized by similar sets of parameters and if these parameters are constant during the evolution. Furthermore, additional processes induced by gravity waves or rotational mixing can interfere with the $s$-process nucleosynthesis. Differences in the $s$-process distributions between the two schemes may be more perceptible in higher mass stars with the LM scheme producing more light $s$-elements. We also report that, contrary to Battino et al. (2016), our simulations do not show evidence for a strong $s$-process nucleosynthesis when using their double exponential overshooting prescription. The reason is ascribed to the fact that with this formulation, mixing is still active in the PM zone during the interpulse phase and allows pollution of the ${ }^{13} \mathrm{C}$-pocket by the ${ }^{14} \mathrm{~N}$ neutron poison.

Acknowledgements. L.S. and S.G. are FRS-F.N.R.S. research associates.

\section{References}

Angulo, C., Arnould, M., Rayet, M., et al. 1999, Nucl. Phys. A, 656, 3 Arnould, M., \& Goriely, S. 2006, Nucl. Phys. A, 777, 157

Asplund, M., Grevesse, N., Sauval, A. J., \& Scott, P. 2009, ARA\&A, 47, 481 Battino, U., Pignatari, M., Ritter, C., et al. 2016, ApJ, 827, 30

Bisterzo, S., Gallino, R., Straniero, O., Cristallo, S., \& Käppeler, F. 2011, MNRAS, 418, 284

Buntain, J. F., Doherty, C. L., Lugaro, M., et al. 2017, MNRAS, 471, 824

Busso, M., Gallino, R., \& Wasserburg, G. 1999, ARA\&A, 37, 239

Chieffi, A., Dominguez, I., Limongi, M., \& Staniero, O. 2001, ApJ, 553, 1159

Cristallo, S., Straniero, O., Gallino, R., et al. 2009, ApJ, 696, 797

De Smedt, K., Van Winckel, H., Kamath, D., et al. 2014, A\&A, 563, L5

De Smedt, K., Van Winckel, H., Kamath, D., et al. 2016, A\&A, 587, A6 Denissenkov, P. A., \& Tout, C. A. 2003, MNRAS, 340, 722 
Freytag, B., Ludwig, H.-G., \& Steffen, M. 1996, A\&A, 313, 497

Goriely, S. 1999, A\&A, 342, 881

Goriely, S., \& Mowlavi, N. 2000, A\&A, 362, 599

Goriely, S., Hilaire, S., \& Koning, A. J. 2008, A\&A, 487, 767

Herwig, F. 2000, A\&A, 360, 952

Herwig, F., Blöcker, T., Schönberner, D., \& Eid, M. E. 1997, A\&A, 324, L81

Herwig, F., Langer, N., \& Lugaro, M. 2003, ApJ, 593, 1056

Herwig, F., Freytag, B., Hueckstaedt, R. M., \& Timmes, F. X. 2006, ApJ, 642, 1057

Herwig, F., Freytag, B., Fuchs, T., et al. 2007, in Why Galaxies Care About AGB Stars: Their Importance as Actors and Probes, eds. F. Kerschbaum, C. Charbonnel, \& R. F. Wing, ASP Conf. Ser., 378, 43

Herwig, F., Woodward, P. R., Lin, P.-H., Knox, M., \& Fryer, C. 2014, ApJ, 792, L3

Iben, I., \& Renzini, A. 1982, A\&A, 263, L23

Käppeler, F., Beer, H., \& Wisshak, K. 1989, Rep. Prog. Phys., 52, 945

Karakas, A. I., \& Lattanzio, J. C. 2014, PASA, 31, e030

Lugaro, M., Karakas, A. I., Stancliffe, R. J., \& Rijs, C. 2012, ApJ, 747, 2

Maeder, A., \& Meynet, G. 1987, A\&A, 182, 243

Marigo, P. 2002, A\&A, 387, 507
Nemeth, Z., Käppeler, F., Theis, C., Belgya, T., \& Yates, S. 1994, ApJ, 426, 357 Nucci, M. C., \& Busso, M. 2014, ApJ, 787, 141

Piersanti, L., Cristallo, S., \& Straniero, O. 2013, ApJ, 774, 98

Reimers, D. 1975, Mem. Soc. Roy. Sci. Liège, 8, 369

Sallaska, A. L., Iliadis, C., Champagne, A. E., et al. 2013, ApJS, 207, 18

Siess, L. 2006, A\&A, 448, 717

Siess, L., Dufour, E., \& Forestini, M. 2000, A\&A, 358, 593

Siess, L., Goriely, S., \& Langer, N. 2004, A\&A, 415, 1089

Sparks, W., \& Endal, A. 1980, ApJ, 237, 130

Stancliffe, R. J., Dearborn, D. S. P., Lattanzio, J. C., Heap, S. A., \& Campbell, S. W. 2011, ApJ, 742, 121

Straniero, O., Gallino, R., \& Cristallo, S. 2006, Nucl. Phys. A, 777, 311

Takahashi, K., \& Yokoi, K. 1987, At. Data Nucl. Data Tables, 36, 375

Van Eck, S., Goriely, S., Jorissen, A., \& Plez, B. 2001, Nature, 793

Vassiliadis, E., \& Wood, P. R. 1993, ApJ, 413, 641

Xu, Y., Goriely, S., Jorissen, A., Chen, G., \& Arnould, M. 2013a, A\&A, 549, A10

Xu, Y., Takahashi, K., Goriely, S., et al. 2013b, Nucl. Phys. A, 918, 61

Zamora, O., Abia, C., Plez, B., Domínguez, I., \& Cristallo, S. 2009, A\&A, 508, 909 